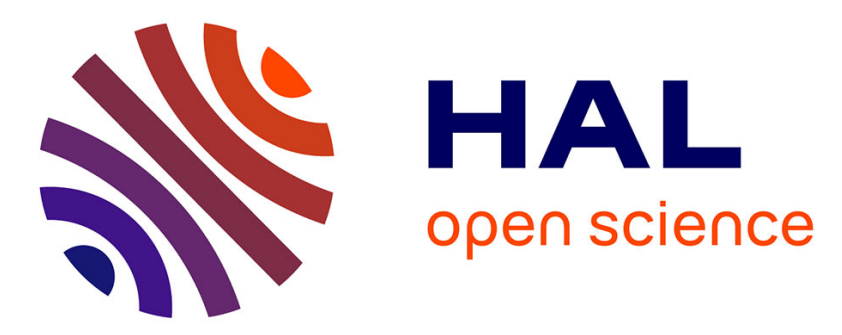

\title{
Choosing a TCP Version over Static Ad Hoc Wireless Networks: Wired TCP or Wireless TCP?
}

\author{
Ivan Marino Martinez Bolivar, Victor Ramos
}

\section{To cite this version:}

Ivan Marino Martinez Bolivar, Victor Ramos. Choosing a TCP Version over Static Ad Hoc Wireless Networks: Wired TCP or Wireless TCP?. 2013 Seventh International Conference on Next Generation Mobile Apps, Services and Technologies (NGMAST), Sep 2013, Prague, Czech Republic. pp.170-174, 10.1109/NGMAST.2013.38 . hal-02300970

\section{HAL Id: hal-02300970 https://hal.science/hal-02300970}

Submitted on 30 Sep 2019

HAL is a multi-disciplinary open access archive for the deposit and dissemination of scientific research documents, whether they are published or not. The documents may come from teaching and research institutions in France or abroad, or from public or private research centers.
L'archive ouverte pluridisciplinaire HAL, est destinée au dépôt et à la diffusion de documents scientifiques de niveau recherche, publiés ou non, émanant des établissements d'enseignement et de recherche français ou étrangers, des laboratoires publics ou privés. 


\section{Choosing a TCP version over static ad hoc wireless networks: wired TCP or wireless TCP?}

\author{
Ivan Martinez \\ Universidad Santo Tomás (USTA) \\ Telecommunications Engineering Faculty \\ Bogotá, Colombia \\ ivanmartinez@usantotomas.edu.co
}

\author{
Victor Ramos \\ Universidad Autónoma Metropolitana (UAM) \\ Department of Electrical Engineering \\ Networking and Telecommunications Research Team \\ Iztapalapa, Mexico City \\ vicman@xanum.uam.mx
}

\begin{abstract}
The Transmission Control Protocol (TCP) was originally designed to operate on wired networks. However, nowadays the traffic on wireless networks has grown to a point where one must take into account the specific characteristics of such networks when setting up a particular TCP implementation on them. Particularly, the TCP performance has been studied over ad hoc wireless networks leading to several new implementations for TCP. The main issue for TCP on ad hoc wireless networks is to differentiate between losses due to congestion and losses occurred at lower network layers. In this paper, we analyze the TCP performance on two different scenarios of static ad hoc wireless networks over the DSDV routing protocol. Our findings show that choosing the right TCP version for ad hoc wireless networks is a key factor for their performance. We show for the scenarios we study, that TCP Reno outperforms TCP Westwood on the average loss rate as well as on the throughput.
\end{abstract}

Keywords - Ad hoc wireless networks; TCP Reno; TCP Westwood; DSDV.

\section{INTRODUCTION}

Unlike traditional wireless networks that are provided with a centralized infrastructure, wireless ad hoc networks are formed solely of a set of nodes with identical or similar characteristics that perform simultaneously functions of a station as well as of a router [1]. These characteristics make ad hoc networks very attractive in terms of cost, scalability, and ease of implementation and thus making them well suited for a wide set of applications as in wireless sensor networks (WSN), emergency situation applications, monitoring of specific locations, mobile object tracking, location characterization, characterizing places, and military applications just to mention a few. Figure 1 depicts a simple example of an ad hoc network.

The deployment of wireless protocols such as Bluetooth, IEEE 802.11, and Hyperlan makes possible the implementation of ad hoc networks for commercial purposes. Therefore, several research efforts have been made during the last decade to solve the main problems faced by this type of network architectures. Wireless ad hoc networks may be classified as mobile ad hoc networks (MANETs) and static ad hoc networks (SANETs). On one hand, in MANETs nodes are allowed to move within a given area, and thus mobility introduces

This work has been done during the Ivan Marino's spring research internship at the Networking and Telecommunications Research Team of UAM-I in Mexico City.

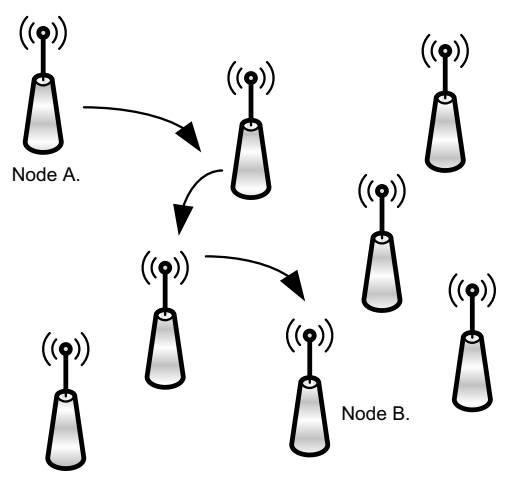

Figure 1. Example of an ad hoc network

additional challenges to overpass for the correct operation of the network. On the other hand, wireless nodes in a SANET are placed within a given area and do not move.

The main problems faced by ad hoc networks include the hidden terminal problem, the exposed terminal problem, path asymmetry, network partition, routing problems, and power constraints. Additionally, in order that applications running over ad hoc networks operate well, a transport layer protocol is often needed. Therefore, several research efforts have been done on the direction of evaluating the performance of the transmission control protocol (TCP) on ad hoc networks [2][4].

The TCP protocol was originally designed to operate over wired networks. However, the wireless nature of ad hoc networks adds new problems to the operation of TCP. The TCP protocol interprets any packet loss as a sign of congestion; however, wireless networks may induce losses due to interference or collisions, which may be badly interpreted by TCP and thus impacting its performance. This is why several versions of TCP for wireless networks have been proposed in the literature to circumvent the deficiencies of TCP when losses other than those due to congestion take place within a TCP connection.

In this work, we compare the performance of two different versions of TCP on a SANET: TCP Reno and TCP Westwood; 
the former being originally designed for wired networks and the latter designed to operate on wired as well as on wireless networks. Our aim is to study the performance of the TCP protocol over SANETs arranged in a mesh, which is a classical node placement for several practical applications. The results we obtain during our analysis show that even if TCP Westwood is designed to operate on wired as well as on wireless networks, it is not always the best choice when selecting a transport protocol for the type of wireless networks as those we consider in this paper, because TCP Reno exhibits a better performance for every case we study.

The remainder of this paper is as follows. In Section II, we briefly describe the background and related work of TCP over ad hoc networks. Section III presents the simulation scenarios we consider for our evaluation. In Section IV, we describe the results we obtain by comparing TCP Reno and TCP Westwood regarding the throughput and the loss rate over our scenarios. Finally, Section V sketches our conclusions.

\section{BACKGROUND AND RELATED WORK}

Several research has been done on TCP over SANETs, the main efforts have been done on how TCP may be well tuned to operate on multi-hop wireless SANETs. In [5], the authors compare the performance of five different TCP implementations: Tahoe, Reno, New Reno, Sack, and Vegas over a multihop network when using the 802.11 protocol; by adequately tunning the TCP's advertised window the authors find that TCP Vegas outperform the other TCP implementations being besides the fairer TCP version. Moreover, the same author shows in [6] that even in scenarios where TCP connections cross wired as well as wireless networks, the TCP unfairness problem persists.

Later, in [7] the authors analyze the effect of the TCP's congestion window limit on throughput for SANETs. They also focus on a multi-hop scenario for a TCP connection and find that the MAC layer has a big impact on TCP performance. Thus, most of the work on TCP over SANETs is focused on how to well tune TCP to operate well on such kind of networks as well as on facing the problem of how to differentiate between losses due to congestion and losses due to phenomena on lower protocol layers. Therefore, several new versions of TCP for wireless networks have been proposed to date $[3,8,9]$ in order to circumvent such TCP deficiencies.

The main conclusion is that, when implementing an application over an ad hoc network it is often better to choose a TCP version for wireless networks rather than one for wired networks. In this paper, we show that this is not always the best choice. We compare two different TCP implementations: Reno and Westwood. The latter has been proposed to operate on wired as well as on wireless networks, while the former is designed to operate on wired networks. By means of simulation with the NS-2 simulator, we find in the following sections that TCP Reno outperforms better than Westwood for all of the cases we study on two different SANET scenarios. The reader is referred to $[4,10]$ for complementary analyses regarding MANETs.

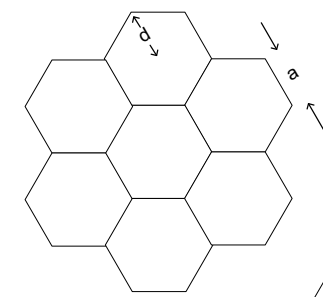

(a)

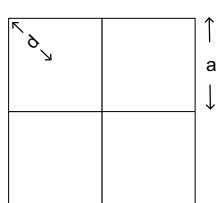

(b)

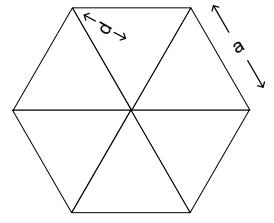

(c)
Figure 2. Most used topologies for static ad hoc networks: (a) Hexagon, (b) Square, and (c) Triangle.

\section{A. Node placement on a SANET}

The node placement on a SANET is choice that depends on the application. Most of the applications for SANETs place nodes arranged in a way such that they cover a whole surface of interest. The most widely used node arrangement is a mesh, but different topologies have been proposed which may better adapted to a particular range of applications [11].

We show in Figure 2 an example of different topologies for mesh deployment in static ad hoc networks. We can find in the literature several application examples that use different node topologies. For instance, in $[12,13]$ the authors implement networks with smoke sensors for early fire detection.

The nodes in our analysis are placed in a square mesh with $20 \mathrm{~m}$ of separation between nodes. We analyze two different scenarios, one with a TCP connection traversing the whole network in order to stress the routing protocol by having the highest number of hops, and the other one with several simultaneous TCP connections sending data to a receiver located at the center of the mesh.

\section{SiMULATION SCENARIOS AND PERFORMANCE MEASURES}

A wide set of applications benefit from placing nodes arranged as a mesh, we thus choose such scenario for evaluation. In [11], the authors provide an analytical model allowing to represent such scenario as the one shown in Figure 2(b), their model is represented as follows:

$$
\begin{gathered}
d=0.71 \times \sqrt{\frac{|A|}{N}} \\
a=\sqrt{\frac{|A|}{N}} \\
N_{s}=0.50 \times \frac{|A|}{d^{2}}
\end{gathered}
$$




$$
N_{t}=\frac{|A|}{a^{2}}
$$

where $n$ represents the maximum allowed distance between any point from the deployment zone and the nearest sensor, $a$ is the length of a tile's side, $N$ is the number of nodes, and $|A|$ is the total area to cover.

Depending of the type of zone to cover and the type of sensors, there is an additional parameter, $\delta$, representing the sampling distance which must accomplish $\delta \geq d$. In order to guarantee the optimal communication among the whole set of nodes, the transmission range $r$ should fulfill $r \geq a$.

We choose the IEEE 802.11 standard for our simulations since it is the most widely used to deploy wireless networks, it is also easily scalable and cost convenient. Additionally, the 802.11 transmission speeds are also according to those used in WSNs, and finally the indoor coverage radio for this standard is $38 \mathrm{~m}$.

We focus our attention on a set of applications benefiting from implementing the scenario chosen. Thus, several WSN implementations where the main application is forest monitoring to warn from fire of from illegal logging, where the $\delta$ parameter depends of the coverage radio of each node $[14,15]$. We thus take these considerations into account and set this value to $\delta=20 \mathrm{~m}$. Finally, the surface were we deploy the set of nodes is a $160 \mathrm{~m} \times 160 \mathrm{~m}$ square, which results in the set of parameters given in Table I

Table I

PARAMETERS FOR THE NETWORK MODEL.

\begin{tabular}{|c|c|}
\hline$A$ & $25600 \mathrm{~m}^{2}$ \\
\hline$a$ & $20 \mathrm{~m}$ \\
\hline$d$ & $14.2 \mathrm{~m}$ \\
\hline$N$ & 64 \\
\hline
\end{tabular}

We therefore consider the two following scenarios. The first one consisting of a network with 64 static wireless nodes arranged in a mesh, where nodes are spaced by $20 \mathrm{~m}$. There is a TCP connection between the end nodes in a manner such that we are able to evaluate a scenario where the connection traverses the maximum number of hops, and thus stressing the routing protocol to find out the path with the best conditions. Figure 3 represents the first scenario.

We consider the same node mesh arrangement for the second scenario, where we set up 64 simultaneous TCP connections between each node and the one located at the center, which acts as receiver. This scenario is closer to a wireless static sensors network characterizing a real static WSN. We show such zone definition in Figure 4.

We use the NS-2 network simulator for our evaluation. Table II lists the parameters we consider for our scenarios. For each case, we run the simulation 1000 times computing average values for throughput as well as for losses.

\section{Results}

We present in this section the results for both scenarios, where we measure the throughput as well as the loss rate for

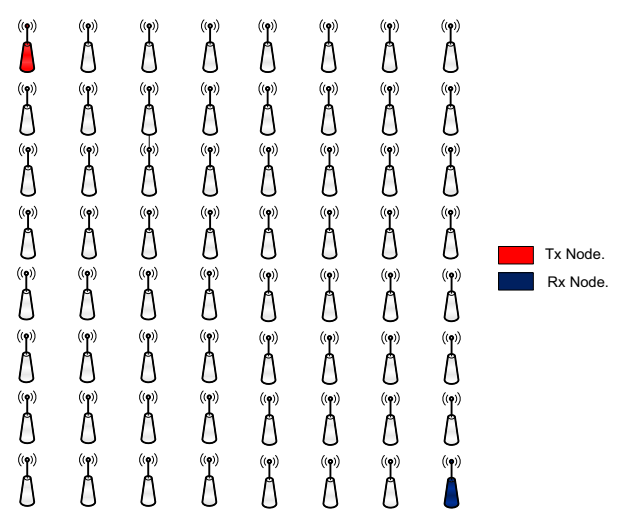

Figure 3. Our first scenario.

Table II

NS-2 SIMULATION PARAMETERS.

\begin{tabular}{|c|c|}
\hline Propagation model & Two-ray ground \\
\hline Distance between nodes & $20 \mathrm{~m}$ \\
\hline Routing protocol & DSDV \\
\hline Surface & $160 \mathrm{~m} * 160 \mathrm{~m}$ \\
\hline Simulation time & $1000 \mathrm{~s}$ \\
\hline Transport protocols & TCP Reno and TCP Westwood \\
\hline Coverage ratio per node & $22 \mathrm{~m}$ \\
\hline Mobile nodes & No \\
\hline
\end{tabular}

each TCP version. The Reno and Westwood versions of TCP are compared over the DSDV routing protocol. We choose DSDV as the routing protocol since given the static nature of our wireless scenarios a proactive routing protocol like DSDV may perform better than a reactive one. Therefore for a network with static nodes, like the one we are considering, a proactive protocol often behaves better compared with a reactive protocol like AODV.

Thus, we show in Figures 5 and 6 the results corresponding

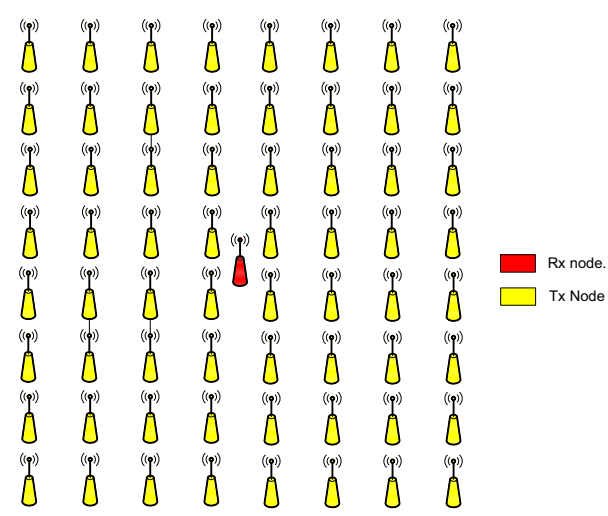

Figure 4. Our second scenario. 


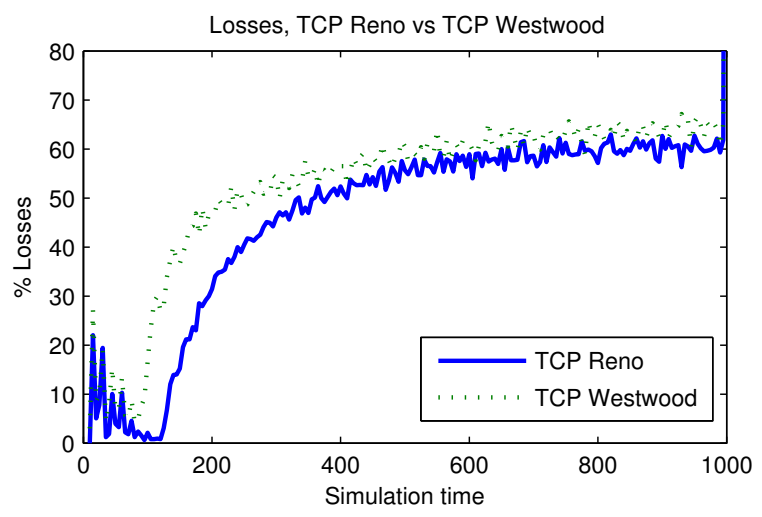

Figure 5. Loss rate for the first scenario.

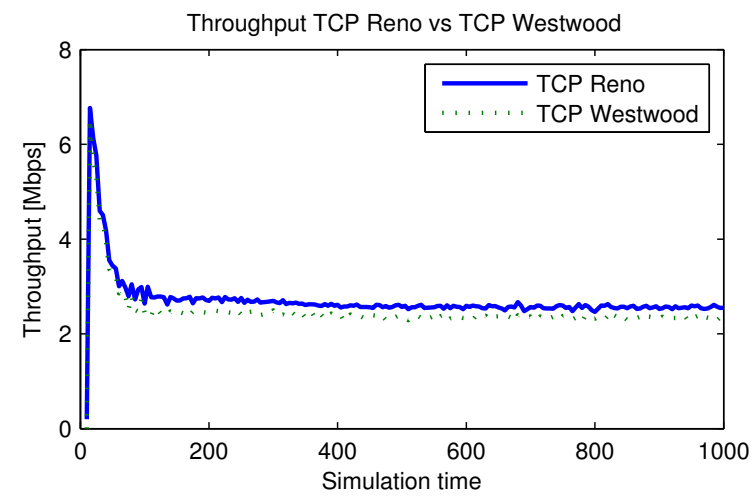

Figure 6. Throughput for the first scenario.

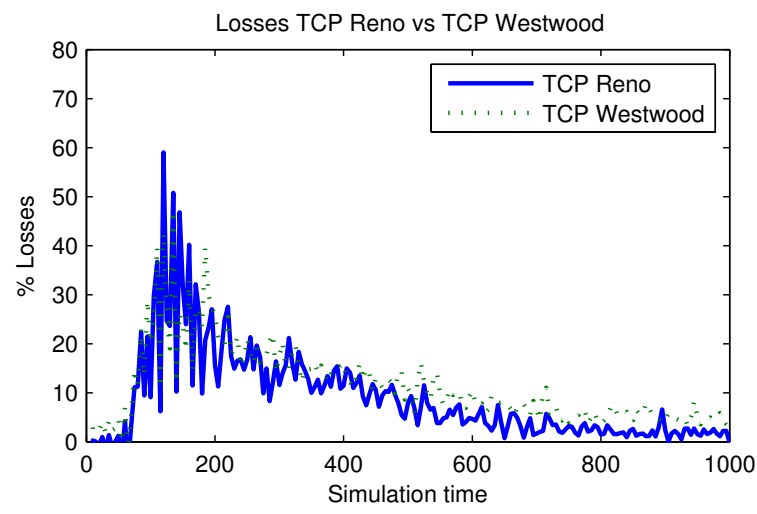

Figure 7. Loss rate for the second scenario.

to the first scenario and in Figures 7 and 8 the results corresponding to the second scenario.

Regarding our first scenario, we clearly see how in Figures 5 and 6 TCP Reno outperforms TCP Westwood regarding the loss rate as well as the throughput. This may be explained by the fact that even if the nodes involved in the TCP connection are opposite end corners in the mesh, the DSDV routing protocol establishes proactively its routing tables for all nodes leading to the case where most of the losses are due

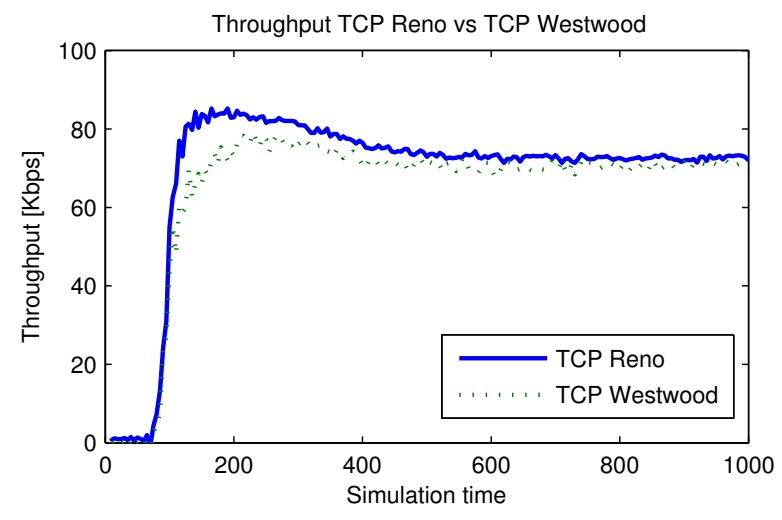

Figure 8. Throughput for the second scenario.

to congestion rather than to other factors (e.g., interference, path asymmetry, route changes). TCP Westwood is particularly designed to better handle large bandwidth-delay product paths (large pipes), considering also losses due to transmission.

Finally, regarding our second scenario we see in Figures 7 and 8 how, again, TCP Reno outperforms TCP Westwood even for the case where all nodes are sending data to the center node. We note how in this case, the loss rate grows considerably compared to the previous scenario which is clearly explained by the fact that the whole set of nodes is active during transmission. In the case of throughput, we measure the average throughput at the center node, that is why the average throughput is considerably lower than for the first scenario.

\section{CONCLusions}

In this paper, we evaluated the performance of TCP Reno and TCP Westwood over a SANET arranged in a mesh topology. Two scenarios have been analyzed, the first one allowed us to evaluate the network performance for the nodes located at extreme corners of the mesh. In this case, the routing protocol makes the TCP connection traverse the higher number of hops within this topology.

The second scenario we studied was intended to evaluate the network performance in the presence of several active TCP connections. Our results for both scenarios reveal that choosing a TCP implementation for wireless networks might not always be the better choice for SANETs since during our evaluations TCP Reno behave slightly better than TCP Westwood.

\section{REFERENCES}

[1] D. Wei, "Analysis of the applications and characteristics of ad hoc networks," in Proceedings of the International Conference on Communication Technology (ICCT), Nov. 2006

[2] A. Al-Hanbali, E. Altman, and P. Nain, "A survey of TCP over ad hoc networks," IEEE Communications Surveys and Tutorials, vol. 7, no. 3, pp. 22-36, Third quarter 2005.

[3] C.-P. Fu and S. Lieu, "TCP Veno: TCP enhancement for transmission over wireless access networks," IEEE Journal on Selected Areas in Communications (JSAC), vol. 21, no. 2, pp. 216-228, 2003. 
[4] S. Henna, "A throughput analysis of TCP variants in mobile wireless networks," in Proceedings of the Third International Conference on Next Generation Mobile Applications, Services and Technologies, Sep. 2009.

[5] S. Xu and T. Saadawi, "Performance evaluation of TCP algorithms in multi-hop wireless packet networks," Journal of Wireless Communications and Mobile Computing, vol. 2, no. 1, pp. 85-100, 2002.

[6] K. Xu, S. Bae, and M. Gerla, "TCP behavior across multihop wireless networks and the wired Internet," in Proceedings of the ACM Workshop on Wireless Mobile Multimedia, Sep. 2002, pp. 41-48.

[7] K. Chen, Y. Xue, and K. Nahrstedt, "On setting TCP's congestion window limit in mobile ad hoc networks," in Proceedings of the IEEE International Conference on Communications (ICC), May 2003.

[8] R. Wang, M. Valla, M. Sanadidi, and M. Gerla, "Adaptive bandwidth share estimation in TCP westwood," in Proceedings of the IEEE Globecom, Nov. 2002.

[9] I. Akyldiz, X. Zhang, and J. Fang, "TCP-Peach+: enhancement of TCPPeach for satellite IP networks," IEEE Communications Letters, vol. 6 , no. 7, pp. 303-305, 2002.

[10] V. Bhanumathi and R. Dhanasekaran, "TCP Variants a comparative analysis for high bandwidth-delay product in mobile ad hoc network," in The 2nd International Conference on Computer and Automation Engineering (ICCAE), Singapore, Feb. 2010, pp. 600-604.

[11] E. Biagioni and G. Sasaki, "Wireless sensor placement for reliable and efficient data collection," in Proceedings of the 36th Annual Hawaii International Conference on System Sciences (HICSSJ Jan. 2003.

[12] Z. Kovács, G. Marosy, and G. Horváth, "Case study of a simple , low power WSN implementation for forest monitoring 2 overview of WSN systems," in Proceedings of 12th Biennial Baltic Electronics Conference (BEC), Oct. 2010, pp. 161-164.

[13] F. Al-Turjman, H. Hossam, and M. Ibnkahla, "Connectivity optimization for wireless sensor networks applied to forest monitoring," iRroceedings of the IEEE International Conference on Communications (ICC) Dresden, Germany, Jun. 2009, pp. 1-6.

[14] Z. Lei and J. Lu, "Distributed coverage of forest fire border based on WSN," in Proceedings of the 2nd International Conference on Industrial and Information Systems, Jul. 2010, pp. 341-344.

[15] J. Papán and J. Jurecka, "WSN for forest monitoring to prevent illegal logging," in Proceedings of the Federated Conference on Computer Science and Information Systems (FedCSIS), Sep. 2012, pp. 809-812. 